\title{
Diabetic striatopathy- rare case of Chorea associated with Non-ketotic hyperglycemia
}

\author{
Moomin Hussain Bhat, Shivani Sidana, Shariq Rashid Masoodi, Atul Sharma \\ Department of Endocrinology, Sher-i-Kashmir Institute of Medical Sciences, Srinagar
}

A B S T RA C T

\begin{abstract}
Non-ketotic hyperglycemia (NKHG) has been reported to increase the probability of seizures and movement disorders especially in elderly population. Here we report a case of long standing diabetes presenting with choreoathetoid movements and poor glycemic control. They may get frequently misdiagnosed to have neurological disease, however recognition of this association is important as the condition may quickly resolve when NKHG is controlled.

Keywords: diabetic striaopathy, hyperglycemia

JMS: 2019; 22(2):42-44ＤOl: https://doi.org/10.33883/jms.v22i3.483
\end{abstract}

\section{INTRODUCTION}

Hyperkinetic movement disorders are characterized by involuntary movements not associated with weakness. Several types of hyperkinetic disorders have been described like tremors, chorea, hemiballismus, athetosis, tics and dystonia. Chorea is a rare movement disorder that can be familial or seen to be associated with a number of other disorders $^{1}$. Association of chorea with vascular diseases, metabolic derangements like hypoglycemia ${ }^{2}$ and hyperglycemia ${ }^{3,4}$ has been described in literature, though rare. Chorea associated with non-ketotic hyperglycemia (NKHG) and high signal intensity lesions on T1-weighted brain magnetic resonance images (MRI) is recognized as a unique syndrome, known as diabetic striatopathy. This has been mainly described to affect elderly women ${ }^{3}$. However, its overall clinical features and pathogenesis are still unclear. Here we describe a case of this unique syndrome in an elderly male who presented with choreoathetoid movements and NKHG.

\section{CASE REPORT}

$55 \mathrm{y} / \mathrm{M}$, plumber by occupation, known case of T2 DM for 5 years, complicated by tripathy presented with history of sudden onset choreoathetoid movements right hand and

\begin{tabular}{|l|l|} 
& Access this article online \\
& Website: \\
&
\end{tabular}

right angle of mouth for 10 days. These involuntary movements were present even during sleep. There was no history suggestive of connective tissue disorders, fever, use of anticonvulsants, cocaine, CNS stimulants or lithium. There was no family history of movement disorder. On examination there were uncontrollable slow, distal, writhing, involuntary, semi-purposeful choreoathetoid movements involving right arm and hand with some involuntary chewing movements involving right angle of mouth, however gait was normal. His muscle tone, power, reflexes and sensory examination was normal. Systemic examination was unremarkable. Investigations revealed random blood glucose of $468 \mathrm{mg} / \mathrm{dl}$, HbA1c- $9.9 \%$, serum sodium $134 \mathrm{mmol} / 1$, calculated serum osmolality $294 \mathrm{mosm} / \mathrm{kg}$, serum ketones were negative normal venous blood gas analysis and thyroid function test.Serum creatinine- $1.8 \mathrm{mg} / \mathrm{dl}$, eGFR-41 $\mathrm{ml} / \mathrm{min} / 1.73 \mathrm{~m}^{2}$, normal hemogram, peripheral blood film and liver function tests. MRI brain was done which revealed T1 hyperintensity, T2

Correspondence:

Dr. Shivani Sidana, MD

Senior resident, Department of Endocrinology, SKIMS, Srinagar. E-mail: shivani66sidana@gmail.com.

How to cite this article: Bhat MH, Sidana S, Masoodi SR, Sharma A. Diabetic striatopathy- rare case of Chorea associated with Non-ketotic hyperglycemia. jms 2019;22(2):42-44

Received:21-11-2019 Accepted: 10-12-2019 
Bhat MH; et al; Diabetic striatopathy- rare case of Chorea associated with Non-ketotic hyperglycemia

hypointensity in left basal ganglia region suggestive of diabetic striatopathy. Keeping in mind his clinical features, NKHG and characteristic MRI features, diagnosis of diabetic striatopathy was made. Hyperglycemia was managed with insulin therapy- basal bolus regimen. Besides insulin therapy, patient was started on tetrabenazine with marked improvement of clinical features within 3 days of start of treatment. Repeat MRI brain was planned after 3 months to look for resolution of brain lesion.

\section{DISCUSSION}

Choreoathetosis, is a rarely described entity, typically seen in elderly women with T2DM and NKHG. However it has been also reported in patients with T1DM children ${ }^{5}$ and those with diabetic ketoacidosis ${ }^{6}$. It is characterized by high signal intensity T1weighted MRI confined to basal ganglia ${ }^{7}$ ${ }^{11}$. Initially these lesions were attributed to be due to postanoxic ischemic calcifications; frequent disappearance of lesion on follow-up suggested it to represent petechial hemorrhage rather than calcification. A biopsy and postmortem pathological studies have shown selective neuronal loss, gliosis, and reactive astrocytosis at the striatal areas, although, no apparent hemorrhage was reported ${ }^{7,10}$. Precise mechanism of diabetic striatopathy is unknown, various causative hypothesis have been postulated. In hyperglycemia, the cerebral metabolism shifts to the anaerobic pathway leading to increase metabolism of GABA, and decreased regeneration of acetate leading to decreased acetylcholine synthesis. A reduction in both GABA and acetylcholine in the basal ganglia and the lack of energy production may then cause a basal ganglia dysfunction and subsequent chorea.". Other postulated mechanism is that poorly controlled diabetes mellitus increases the risk of cerebrovascular ischemia and reduces cerebral blood flow ${ }^{12}$. Combined effects of neurotransmitter deregulation, hyperglycemia and vascular insufficiencies cause an incomplete and transient dysfunction of the basal ganglia clinically manifesting as chorea.

Radiologically, lesion is characterized by characteristic hyper-intense basal ganglia lesions in T1-weighted brain MRI, but the T2-weighted brain MRI findings vary. High signal intensity in basal ganglia region on the T1-weighted MRI studies is not specific to diabetic striatopathy and can be observed in chronic hepatic encephalopathy ${ }^{13}$, postcardiac arrest encephalopathy ${ }^{14}$, hypoglycemic coma ${ }^{15}$, and mild focal ischemia ${ }^{16}$.

Patient usually respond well after management of hyperglycemia with insulin therapy, with variable time to response from one day to months ${ }^{17,18}$. Some patients need other medications temporarily to control involuntary movements. But overall prognosis has been reported to be excellent ${ }^{17-20}$, and only few atypical cases with delayed onset after resolution of the hyperglycemia, unremitting severe disorder movements, and late recurrence have been reported $^{8,9}$.

To conclude diabetic striatopathy as a rare cause of chorea with characteristic T1 hyper-intense lesion in basal ganglia region occurs in elderly women with longstanding and poorly controlled diabetes, but not exclusively. Its clinical presentation is variable course is benign, and most patients have transient movement disorders that respond well to medical treatment as evident with resolution of clinical and radiological features. Thus, diabetic striatopathy should be kept in mind especially while managing an elderly patient of movement disorder and underlying longstanding uncontrolled diabetes and typical lesion on MRI, due to good prognosis of disease if managed properly.

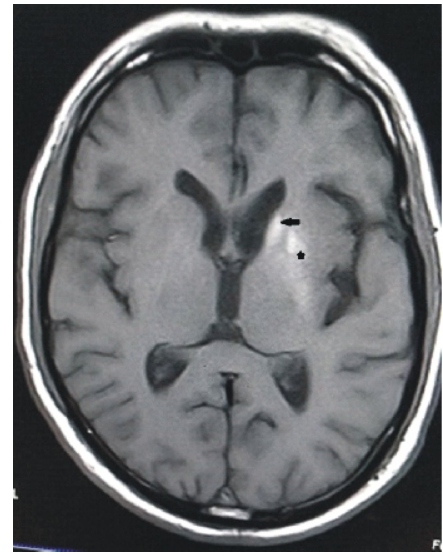

Fig 1a: T1W MRI showing hyperintensity at caudate nucleus (Arrow) and lentiform nucleus (Star)

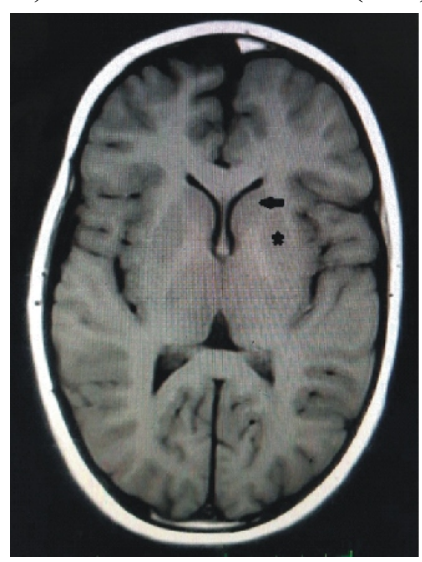

Fig 1b:T1W MRI showing normal hypointense caudate nucleus (Arrow) and lentiform nucleus (Star) Comparative image. 
Bhat MH; et al; Diabetic striatopathy- rare case of Chorea associated with Non-ketotic hyperglycemia

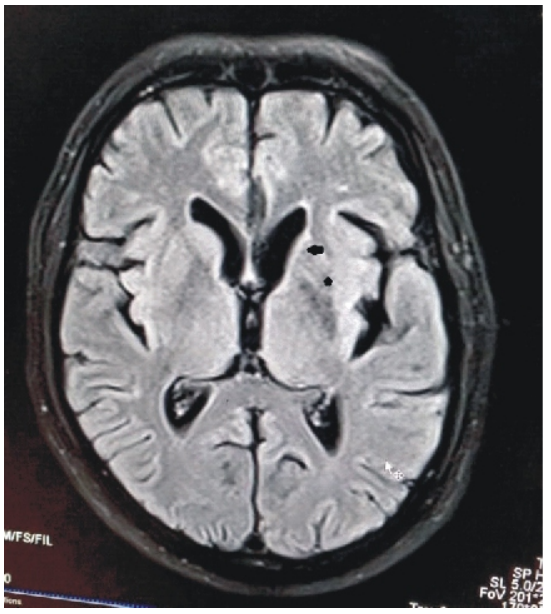

Fig 2a: FLAIR MRI showing isointensity at caudate nucleus (Arrow) and iso/hypointense lentiform nucleus (Star)

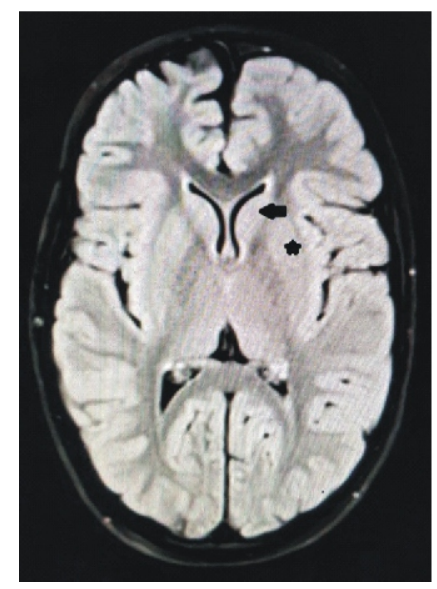

Fig 2b:FLAIR MRI showing normal hyperintense caudate nucleus (Arrow) and lentiform nucleus (Star) Comparative image.

\section{REFERENCES}

1. Kasper DL, Fauci AS, Hauser S, et al, editors. Harrison's principles of internal medicine, 19th ed.New York: The McGraw-Hill Companies, Inc.; 2015: 2618-23.

2. Newman RP, Kinkel WR. Paroxysmal choreoathetosis due to hypoglycemia. Arch Neurol 1984;41:341-2.

3. LeeBC, HwangSH, ChangGY. Hemiballismus hemichorea in older diabetic women: a clinical syndrome with MRI correlation. Neurology 1999;52:646-8.

4. Lai PH, Tien RD, Chang MH et al. Chorea-ballismus with nonketotic hyperglycemia in primary diabetes mellitus. AJNR Am J Neuroradiol 1996; 17(6): 1057-64.

5. Faundez $T$, Klee $P$, Hanquinet $S$ et al. Diabetic striatopathy in childhood. A case report. Pediatrics 2016; 137.

6. Nabatame H, Nakamura K, Matsuda M, Fujimoto N, Shio
H. Hemi- chorea in hyperglycemia associated with increased blood flow in the contralateral striatum and thalamus. Int Med 1994;33:472 5.

7. Shan DE, Ho DM, Chang $\mathrm{C}$ et al. Hemichorea hemiballism: an explanation for MR signal changes. Am J Neuroradiol 1998;19:863 70.

8. Postuma RB, Lang AE. Hemiballism: revisiting a classic disorder. Lancet Neurol 2003; 2(11): 661-8.

9. El Otmani H, Moutaouakil $\mathrm{F}$ et al. Chorea-ballismus in acute non- ketotic hyperglycaemia. FunctNeurol 2009; 24(3): 129-32.

10. Ohara S, Nakagawa $S$ et al. Hemiballism with hyperglycemia and striatal T1-MRI hyperintensity: an autopsy report. MovDisord 2001; 16(3): 521-5.

11. Guisado R, Arieff AI. Neurological manifestations of diabetic comas: correlation with biochemical alterations in the brain. Metabolism 1975;24:665-79.

12. Oh SH, Lee KY et al. Chorea associated with non-ketotic hyperglycemia and hyperintensity basal ganglia lesion on T1-weighted brain MRI study: a meta-analysis of 53 cases including four present cases Journal of the Neurological Sciences 200 (2002) 57-62.

13. Krieger D, Krieger S, Jansen O et al. Manganese and chronic hepatic encephalopathy. Lancet 1995;346:270-4.

14. Fujioka M, Okuchi K, Sakaki T, Hiramatsu K et al. Specific changes in human brain following reperfusion after cardiac arrest. Stroke 1994;25:2091-5.

15. Fujioka M,Okuchi K,Hiramatsu K et al. Specific changes in human brain after hypoglycemic injury. Stroke 1997;28: 584-7.

16. Mirowitz SA, Westrich T, Hirsh JD. Hyperintense basal ganglia on T1-weighted MR images in patients receiving parental nutrition. Ra- diology 1991;181:117-20.

17. Younes S, Cherif Y, Aissi M, Alaya W, Berriche O, Boughammoura A, et al. Seizures and movement disorders induced by hyperglycemia without ketosis in elderly. Iran J Neurol. 2014 Jul 4;13(3):172-6.

18. Scherer C. Seizures and non-ketotic hyperglycemia. Presse Med 2005; 34(15): 1084-6. [In French].

19. Lammouchi T, Zoghlami $\mathrm{F}$ et al, Epileptic seizures in non-ketotic hyperglycemia. Neurophysiol Clin 2004; 34(3-4): 183-7.

20. Hennis A, Corbin D, Fraser H. Focal seizures and nonketotic hyperglycaemia. J NeurolNeurosurg Psychiatry. 1992 Mar;55(3):195-7. 